\title{
Pneumocystis and glucocorticoid use: to prophylax or not to prophylax (and when?); that is the question
}

\author{
Kevin L Winthrop, ${ }^{1}$ John W Baddley ${ }^{2}$
}

\begin{abstract}
Pneumocystis jiroveci is an opportunistic fungus with the ability to cause lethal pneumonia in those with advanced immunosuppression. ${ }^{1}$ Fortunately, this outcome is preventable with prophylaxis. Unfortunately, however, deciding who is immunosuppressed enough to justify prophylaxis can be a confusing subject, particularly among rheumatology patients where immunosuppression waxes and wanes based on the use of immunosuppressive therapies and the contribution of the underlying inflammatory disease. Foggy notions persist regarding who is at risk, the level of absolute risk where the risk-benefit of using trimethoprim/sulfamethoxazole (TMP/SMX) or other prophylaxis is worthwhile, and when can prophylaxis be safely stopped. ${ }^{2}$ The article by Park et al published in the Annals of Rheumatic Diseases ${ }^{3}$ sheds light on these important questions, such that a picture of how to approach this issue clinically might finally be more clear for the practicing rheumatologist.
\end{abstract}

\section{WHAT IS THE BENEFIT OF TMP/SMX PROPHYLAXIS?}

Given the difficulty studying these questions in a randomised controlled trial fashion, comparative effectiveness studies such as this one might provide the next best thing. In the article, the authors address the following questions: in patients starting high-dose glucocorticoids and taking them greater than 4 weeks, what is the risk of pneumocystic jiroveci pneumonia (PJP) (and the risk factors for it) and how does risk relate to dose? Further, they evaluate the efficacy of TMP/SMX prophylaxis. To answer these questions, they retrospectively identified an institutional cohort of rheumatology patients in Korea treated with

\footnotetext{
${ }^{1}$ Division of Infectious Diseases, Oregon Health and Sciences University, Portland, Oregon, USA

${ }^{2}$ Division of Infectious Diseases, University of Alabama, Birmingham, Alabama, USA
}

Correspondence to Dr Kevin L Winthrop, Division of Infectious Diseases, Oregon Health and Sciences University, Portland, Oregon 97239-3098, USA; winthrop@ohsu.edu 'high-dose' glucocorticoids ( $\geq 30 \mathrm{mg} /$ day) for 4 or more weeks. Within this cohort, they selected patients offered TMP/SMX prophylaxis and compared their incidence of PJP with the remainder of the group that did not receive prophylaxis. There were important underlying differences between the groups, as one might expect, and it was clear that the treating physicians had generally chosen to give TMP/SMX to those they had perceived at higher risk for PJP. These risk factors included lymphopaenia, greater glucocorticoid use in the past, concomitant use of cyclophosphamide and the presence of dermatomyositis, microscopic polyangiitis (MPA) or granulomatosis polyangiitis. These factors being more prevalent within the prophylaxis group create 'confounding by indication' or 'channelling bias' such that one might expect a higher incidence of PJP in the group receiving prophylaxis, making it difficult to ascertain any protective effect of prophylaxis. This bias is the bane of observational and pharmacoepidemiological studies, as researchers struggle to compare 'apples with apples' and overcome this bias. In this case, the researchers used propensity scores to adjust for differences in groups and created two groups similar in their likelihood to receive prophylaxis based on disease characteristics and underlying risk factors for PJP (those risk factors beyond the use of high-dose glucocorticoids, a risk that was present in all participants). Crude incidence among the unprophylaxed was significantly higher, and when controlling for bias through propensity scores, use of TMP/SMX was associated with a $93 \%$ decrease in incidence of PJP. Only one case occurred in the prophylaxis group, and this after initial TMP/SMX was stopped due to an adverse drug reaction. The protection therefore appeared nearly complete and those at highest risk were protected. The benefits of TMP/SMX cannot be understated, and this study adds to others showing similarly high levels of protective effects within different settings of immunosuppression. ${ }^{4}$ So, we know that it works, but what other conclusions can we draw from this experience that are relevant to the practice of rheumatology?

\section{WHEN TO START PROPHYLAXIS?}

The observations from these researchers confirm the answer is glucocorticoid dose-dependent. This is intuitive, although to our knowledge this has not been shown previously with regard to time to event, in that patients starting on $30 \mathrm{mg}$ dosing took several months longer on average to develop PJP than those who started $60 \mathrm{mg} /$ day. This in part could be related to the fact that patients starting higher doses take much longer to taper below a threshold level of risk. They spend longer times at risk. While Park et al's data suggest you have longer to make a decision regarding

\begin{tabular}{|c|c|c|c|}
\hline \multirow[b]{2}{*}{ Underlying disease } & \multicolumn{2}{|c|}{ Prophylaxis at glucocorticoid dose $(\mathrm{Y} / \mathrm{N}) \dagger$} & \multirow{2}{*}{$\begin{array}{l}\text { Discontinuation } \\
\text { of prophylaxis at } \\
\text { glucocorticoid dose }(\mathrm{Y} / \mathrm{N}) \\
<15 \mathrm{mg}\end{array}$} \\
\hline & $15-30 \mathrm{mg}$ & $>30 \mathrm{mg}$ & \\
\hline $\begin{array}{l}\text { Granulomatosis with } \\
\text { polyangiitis }\end{array}$ & Y & Y & $Y \ddagger$ \\
\hline Microscopic polyangiitis & Y & $Y$ & $Y \ddagger$ \\
\hline Systemic sclerosis & Y§ & Y & Y \\
\hline $\begin{array}{l}\text { Dermatomyositis/ } \\
\text { polymyositis }\end{array}$ & Y§ & Y & Y \\
\hline $\begin{array}{l}\text { Systemic lupus } \\
\text { erythematosus }\end{array}$ & $\mathrm{N}$ & Y & $Y$ \\
\hline Rheumatoid arthritis & $\mathrm{N}$ & Y§ & Y \\
\hline \multicolumn{4}{|c|}{$\begin{array}{l}\text { *Based on limited data and expert opinion, the authors advocate additional studies to further refine } \\
\text { recommendations in this area. } \\
\text { †Requires prolonged glucocorticoids ( } \geq 4 \text { weeks). } \\
\text { ¥Conditional on }<2 \text { additional risk factors at time of discontinuation: baseline lymphopaenia, low CD4 count, } \\
\text { cyclophosphamide use, anti-TNF or rituximab use, initial glucocorticoid dose of }>60 \mathrm{mg} \text {. } \\
\S \text { Conditional on at least one additional risk factor: baseline lymphopaenia, low CD4 count, cyclophosphamide use, } \\
\text { anti-TNF or rituximab use, initial glucocorticoid dose of }>60 \mathrm{mg} \text {. }\end{array}$} \\
\hline
\end{tabular}


prophylaxis start in those using $30 \mathrm{mg}$ (versus $60 \mathrm{mg}$ for example), it is clear that in any patient where such a dose is envisioned for 4 weeks or longer, that prophylaxis should be initiated.

\section{WHEN TO STOP PROPHYLAXIS?}

The concept of a risk 'threshold' has forever been a 'holy grail' type of question, with initial thresholds drawn at $15 \mathrm{mg}$ or $20 \mathrm{mg}$ per day for greater than 3 weeks. These were based on initial case series data that suggested most cases occur at these dose levels or higher after a prolonged time period. ${ }^{5}$ The current study is consistent with these prior case series and, importantly, highlights that this threshold with regard to dose and time is not uniform. Three $(10 \%)$ of the cases within this series were diagnosed with PJP after tapering to doses below $15 \mathrm{mg} /$ day; however, each case had at least one other risk factor for PJP. We agree with the authors that at this level of glucocorticoid use, the overall risk is much lower $(90 \%$ of the cases occurred at dose levels above $15 \mathrm{mg} /$ day), but these cases illustrate that the risk of a certain dosage of glucocorticoids is likely modified by other PJP risk factors. The benefit of prophylaxis is almost certainly different in an 80 year old with lymphopaenia and vasculitis using $10 \mathrm{mg} /$ day of glucocorticoids as compared with a 50 year old with rheumatoid arthritis (RA) using the same dose who lacks other risk factors. We suggest that for patients receiving prophylaxis that the treating physician consider stopping TMP/SMX once doses have been tapered to $15 \mathrm{mg} /$ day, but that strong consideration be given to continuing it until lower doses are achieved if other PJP risk factors are present.

\section{WHAT IS THE RISK-BENEFIT OF TMP/ SMX?}

The risk-benefit of TMP/SMX prophylaxis has been debated, given the high incidence of side effects reported with this compound. ${ }^{6}$ Prior analyses suggest that the benefit outweighs the risk only in certain inflammatory disease conditions such as vasculitis or dermatomyositis, largely because the frequency of PJP is higher in these conditions. ${ }^{4}$ Notably, prophylaxis may not be favourable in rheumatoid arthritis where the risk is low and number needed to treat (NNT) is much higher. ${ }^{4}$ While this analysis is limited to those using high-dose glucocorticoids for greater than 1 month, it supports these ideas and provides both NNT and numbers needed to harm $(\mathrm{NNH})$ information within some disease subgroups. Overall, this cohort tolerated TMP/SMX fairly well, with approximately $15 \%$ of patients developing AEs attributable to TMP/SMX. This was similar to findings from other analyses of rheumatic disease patients using TMP/SMX prophylaxis. The authors did not report what percentage of those using TMP/SMX withdrew drug due to adverse events; however, the incidence of serious adverse events attributable to TMP/ SMX was low ( $n=2$ events), such that the NNTs in order to prevent one PJP case were lower than the $\mathrm{NNH}$ with regard to serious adverse events. Not surprisingly, this benefit-risk scenario varied by disease state where the NNT was markedly lower for the higher risk diseases such as MPA or systemic lupus erhythematosus (SLE).

There were some important limitations to this analysis. Most PJP cases were diagnosed by use of PCR testing on induced sputum or bronchoalveolar lavage fluid, a situation in which it is sometimes difficult to distinguish between colonisation and definitive PJP. While there should be no differential bias between the two exposure groups that would affect one's ability to judge TMP/SMX effectiveness, the study might overestimate the risk of PJP and hence NNT calculations if some of these cases were only colonisation. It is also unclear if these data can generalise outside of Korea where the prevalence of PJP colonisation (and therefore the risk of developing disease) might differ. In addition, the analysis did not evaluate the risk of biological therapies. It is possible that their use might modify the risk of glucocorticoids, and that this could vary by their mechanism of action. Further, the analysis only addressed risk among a highdose glucocorticoid using population, and it did not report PJP incidence in rheumatology patients who were not using highdose glucocorticoids. The regimen studied was single-strength daily TMP/Sulfa. The risk/benefit of intermittent (three times a week) double strength TMP/SMX may be different, although experience from other settings of immunosuppression would suggest similar efficacy as daily single strength therapy. ${ }^{8}$ Last, it is unclear if prophylaxis with other regimens (eg, dapsone, atovaquone) is of similar utility or should be employed if patients cannot tolerate TMP/SMX.

While these and other clinical questions remain, our opinion is that the current analysis provides strong guidance in terms of who to select for prophylaxis. It supports the efficacy of TMP/ SMX use among those starting regimens of Prednisone $>30 \mathrm{mg} /$ day who continue for greater than 1 month and particularly those starting higher doses such $60 \mathrm{mg} /$ day where the short-term risk of PJP is much greater. This is no matter their underlying disease state, although the benefits of prophylaxis are greater in those with higher risk diseases (eg, vasculitis) and other risk factors. We recommend such prophylaxis should continue until doses are below $15 \mathrm{mg} / \mathrm{day}$, and even at this level if other PJP risk factors such as cyclophosphamide use, lymphopaenia or underlying vasculitis are present (table 1 ). While the absolute risk of PJP is considered to be low within rheumatology, this analysis clearly shows the risk is substantial within certain subgroups of diseases. This is a potentially lethal and preventable infection, and the Park et al analysis suggests there is little reason to take a risk with a month or more of high-dose glucocorticoids.

\section{Handling editor Tore K Kvien}

Contributors KLW and JWB shared all writing responsibilities for the editorial.

Funding This research received no specific grant from any funding agency in the public, commercial or notfor-profit sectors.

Competing interests KLW has been a consultant and/or investigator for Pfizer, AbbVie, Lilly, BMS, Galapagos and UCB. JWB has been a consultant for Pfizer, Merck and R-Pharm.

Provenance and peer review Commissioned; externally peer reviewed.

(C) Article author(s) (or their employer(s) unless otherwise stated in the text of the article) 2018. All rights reserved. No commercial use is permitted unless otherwise expressly granted.

\section{Check for updates}

To cite Winthrop KL, Baddley JW. Ann Rheum Dis 2018;77:631-633

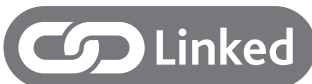

- http://dx.doi.org/10.1136/annrheumdis-2017211796

Ann Rheum Dis 2018;77:631-633.

doi:10.1136/annrheumdis-2017-212588

\section{REFERENCES}

1 Catherinot E, Lanternier F, Bougnoux ME, et al. Pneumocystis jirovecii Pneumonia. Infect Dis Clin North Am 2010;24:107-38.

2 Cettomai D, Gelber AC, Christopher-Stine L. A survey of rheumatologists' practice for prescribing pneumocystis prophylaxis. J Rheumatol 2010;37:792-9.

3 Park JW, Curtis JR, Moon J, et al. Prophylactic effect of trimethoprim-sulfamethoxazole for pneumocystis pneumonia in patients with rheumatic diseases exposed to prolonged high-dose glucocorticoids. Ann Rheum Dis 2018;77:664-9.

4 Green H, Paul M, Vidal L, et al. Prophylaxis of Pneumocystis pneumonia in immunocompromised non-HIV-infected patients: systematic review and metaanalysis of randomized controlled trials. Mayo Clin Proc 2007;82:1052-9. 
5 Yale SH, Limper AH. Pneumocystis carinii pneumonia in patients without acquired immunodeficiency syndrome: associated illnesses and prior corticosteroid therapy. Mayo Clin Proc 1996;71:5-13.

6 Wolfe RM, Peacock JE. Pneumocystis Pneumonia and the Rheumatologist: Which Patients Are At Risk and
How Can PCP Be Prevented? Curr Rheumatol Rep 2017;19:35

7 Rodriguez M, Fishman JA. Prevention of infection due to Pneumocystis spp. in human immunodeficiency virusnegative immunocompromised patients. Clin Microbiol Rev 2004;17:770-82
8 Stern A, Green H, Paul M, et al. Prophylaxis for Pneumocystis pneumonia (PCP) in non-HIV immunocompromised patients. Cochrane Database Syst Rev 2014:Cd005590. 